\title{
Scedosporium apiospermum
}

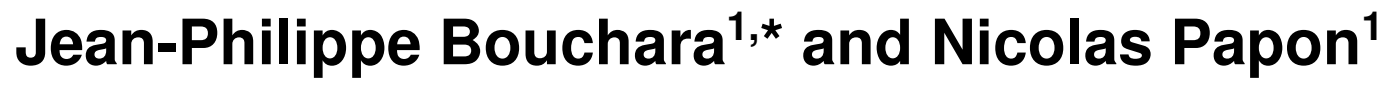

${ }^{1}$ Groupe d'Etude des Interactions Hôte-Pathogène (GEIHP, EA 3142), SFR ICAT 4208, UNIV Angers, UNIV Brest, Angers, France.

* Correspondence: Jean-Philippe.Bouchara@univ-angers.fr (J.-P. Bouchara).

Keywords: Cystic fibrosis; Chronic colonization; Host immune defense evasion; Adaptation

Usually a soil saprophyte, Scedosporium apiospermum is one of the major pathogenic Scedosporium species. Beside chronic localized infections resulting from traumatic inoculation of some soil fungal elements, this cosmopolitan filamentous fungus may also cause respiratory or disseminated infections through inhalation of conidia, particularly in patients with hematological malignancies, solid organ transplant recipients, and patients with cystic fibrosis (CF). With a frequency ranging from 3.5 to $11.7 \%$, Scedosporium species rank second among the filamentous fungi chronically colonizing CF airways. Although well tolerated in most cases, this airway colonization may lead to bronchitis or allergic broncho-pulmonary mycoses, but also to disseminated infections in patients undergoing lung or heart-lung transplantation. Here we summarize the recent advances on the mechanisms allowing the fungus to evade host immune defenses and to establish a chronic respiratory infection.

\section{KEY FACTS:}

A soil fungus that assimilates numerous aromatic or polycyclic hydrocarbons.

A worldwide distribution, but mainly found in human-impacted areas such as industrial areas, city parks, roadsides, and farming lands.

Highly semi-selective culture media are available for detection of Scedosporium species.

First genome sequence of $S$. apiospermum (43.4 Mbp; 10,919 predicted genes) published in 2014.

\section{DISEASE FACTS:}




\section{Trends in Microbiology}

S. apiospermum may cause various human infections ranging from localized infections such as subcutaneous and bone or joint infections, to severe and often fatal disseminated infections in immunocompromised hosts.

This old pathogen has recently gained increasing attention, because of its worldwide recognition as a significant pathogen in CF.

Colonization of CF airways constitutes a major risk factor for a disseminated infection in the case of lung transplantation and all efforts should be made to detect the airway colonization as early as possible and to eradicate the fungus, particularly at registration on the transplantation waiting list.

The limited susceptibility of this fungus to current antifungals justifies work aiming to elucidate its biology and pathogenic mechanisms, which has already led to the identification of some promising targets for drug development, e.g., enzymes involved in uridine synthesis, acylation of inositol, nonribosomal peptide (NRP) synthesis or detoxification of reactive oxygen species.

\section{TAXONOMY AND CLASSIFICATION:}

KINGDOM: Fungi

PHYLUM: Ascomycota

CLASS: Sordariomycetes

ORDER: Microascales

FAMILY: Microascaceae

GENUS: Scedosporium

SPECIES: apiospermum

\section{LITERATURE}

1. Hedayati, M.T. et al. (2019) Fungal epidemiology in cystic fibrosis patients with a special focus on Scedosporium species complex. Microb. Pathog. 129, 168-175

2. Le Govic, Y. et al. (2018) Genomic organization and expression of iron metabolism genes in the emerging pathogenic mold Scedosporium apiospermum. Front. Microbiol. 9, 827

3. Morales, T. et al. (2017) The genomic study of an environmental isolate of Scedosporium apiospermum shows its metabolic potential to degrade hydrocarbons. Stand. Genomic Sci. 12, 71

4. Ramirez-Garcia, A. et al. (2018) Scedosporium and Lomentospora: an updated overview of underrated opportunists. Med. Mycol. 56(suppl_1),102-125

5. Rougeron, A. et al. (2018) Ecology of Scedosporium species: present knowledge and future research. Mycopathologia 183, 185-200

6. Schwarz, C. et al. (2018) Developing collaborative works for faster progress on fungal respiratory infections in cystic fibrosis. Med. Mycol. 56(suppl_1), 42-59

7. Seidel, D. et al. (2019) Prognostic factors in 264 adults with invasive Scedosporium spp. and Lomentospora prolificans infection reported in the literature and FungiScope ${ }^{\circledR}$. Crit. Rev. Microbiol. Jan 10, 1-21 


\section{Trends in Microbiology | Microbe of the Month}

8. Staerck, C. et al. (2019) Transcriptional profiling of Scedosporium apiospermum enzymatic antioxidant gene battery unravels the involvement of thioredoxin reductases against chemical and phagocytic cells oxidative stress. Med. Mycol. 57, 363-373

9. Vandeputte, P. et al. (2014) Draft genome sequence of the pathogenic fungus Scedosporium apiospermum. Genome Announc. 2(5). pii: e00988-14

10. Wiederhold, N.P. (2018) The antifungal arsenal: alternative drugs and future targets. Int. J. Antimicrob. Agents. 51, 333-339 


\section{Scedosporium}

\section{apiospermum}

in the cystic fibrosis context
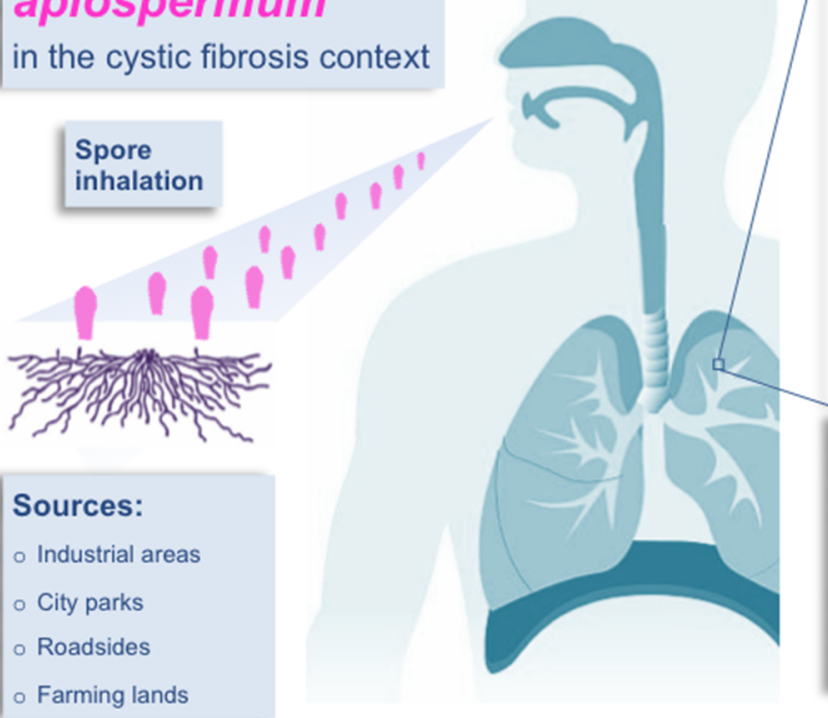

Entrapment

(sticky mucus)

and germination

Adaptation to:

- Hypercapnia

- Hypoxia

- Low osmotic pressure

- Acid pH

- Elevated lactate

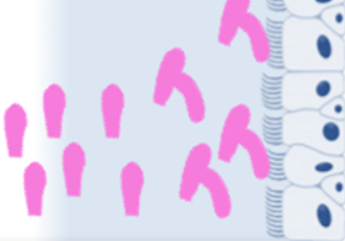

Spore evasion to the host immune defenses

- Dihydroxynapthalene-melanin

- Degradation of reactive oxygen species (glycosyl phosphatidyl inositol-anchored enzymes)

Spore germination inducing

- Biofilm formation

- NRP synthesis

- Polyketide synthesis 


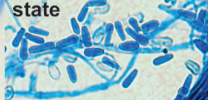

apdiga
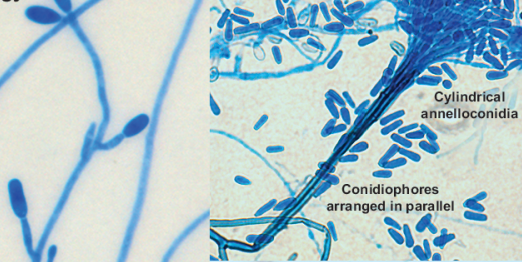

\section{Pseudallescheria}

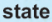

One-celled

ovoid to clavate conidia with a truncated base

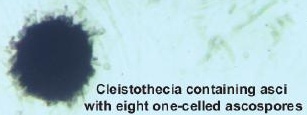

